\title{
Feature Extraction and Classification to Diagnose Hypoxic-Ischemic Encephalopathy Patients by Using Susceptibility-Weighted MRI Images
}

\author{
Sisi $\mathrm{Wu}^{1}$, Sasan Mahmoodi ${ }^{1}$, Angela Darekar ${ }^{2}$, Brigitte Vollmer ${ }^{3}$, \\ Emma Lewis ${ }^{2}$, Maria Liljeroth ${ }^{2}$ \\ 1. Electronics and Computer Science, University of Southampton, University Road, Southamp- \\ ton, Southampton, United Kingdom, SO17 1BJ \\ 2. Department of Medical Physics, University Hospital Southampton NHS Foundation Trust, \\ Tremona Road, Southampton, United Kingdom, SO16 6YD \\ 3. Clinical Neurosciences, Clinical and Experimental Sciences, University of Southampton, \\ Southampton, United Kingdom, SO17 1BJ \\ sw5e12@soton.ac.uk, sm3@ecs.soton.ac.uk
}

\begin{abstract}
In this paper. a method is presented to enable automatic classification of the degree of abnormality of susceptibility-weighted images (SWI) acquired from babies with hypoxic-ischemic encephalopathy (HIE), in order to more accurately predict eventual cognitive and motor outcomes in these infants. SWI images highlight the cerebral venous vasculature and can reflect abnormalities in blood flow and oxygenation, which may be linked to adverse outcomes. A qualitative score based on magnetic resonance imaging (MRI) analyses is assigned to SWIs by specialists to determine the severity of abnormality in an HIE patient. The method allows the detection of image ridges, representing the vessels in SWIs, and the histogram of the ridges grey scales. A curve with only four parameters is fitted to the histograms. These parameters are then used to estimate the SWI abnormality score. The images are classified by using a $k \mathrm{NN}$ and multiple SVM classifiers based on the parameters of the fitting curves. The algorithm is tested on an SWI-MRI dataset consisting of 10 healthy infants and 48 infants with HIE with a range of SWI abnormality scores between 1 and 7 . The accuracy of classifying babies with HIE vs. those without (ie: healthy controls) using our algorithm with a leave-one-out strategy is measured as $91.38 \%$. Our method is fast and could increase the prognostic value of these scans, thereby improving management of the condition, as well as elucidating the disease mechanisms of HIE.
\end{abstract}

Keywords: HIE, SWI, soft ridge detection, histogram, curve fitting, automatic diagnosis. 


\section{Introduction}

Hypoxic-ischemic encephalopathy (HIE) is a type of neurological impairment or injury in infants caused by low oxygen during the perinatal period, and it is a long-term, frequent, severe disease [1-2]. In the developed world, HIE is a common illness affecting approximately 1.5-2 patients per 1,000 new-born infants per year [3]. Infants with moderate encephalopathy have a $10 \%$ risk of death, and those who survive have a $30 \%$ risk of disability. Sixty percent of infants with severe encephalopathy die, and many, if not all, survivors are handicapped [4-5]. Hence, specialists need to diagnose the infant in good time and look at the severity of abnormality. So far, MRI plays an important role in HIE diagnosis. An MR image can confirm a normally developed brain, assess the severity and pattern of any neonatal injury, or even potentially predict the outcome from the pattern of injury and clinical details. Susceptibility weighted imaging (SWI) is a type of MR image; an SWI image highlights the cerebral venous vasculature, reflecting abnormalities in blood flow and oxygenation which may be linked to adverse outcomes. A qualitative score based on MRI analyses can be assigned to SWIs by specialists to provide an assessment of the degree of severity of an abnormality in an HIE patient [19].

In previous research, numerous studies have focused on the cerebral vessel structure and vessel detection. In the medical research, a number of studies on HIE diagnosis have focused on Diffusion Weighted Imaging (DWI) [6-7, 21] and Electroencephalogram (EEG) diagnosis [8-9]. In [21], DWIs are acquired for the apparent diffusion coefficient (ADC) map to evaluate the quantification of ischemic injury by employing the watershed and superpixel methods. This is considered the first step of diagnosis in which the 2D slices of DWIs are inspected. The main issue in the work [21] is that the number of abnormal cases is limited to three patients. However the score predicted by the method proposed in [3] has a 0.84 correlation coefficient. The other type of MRIs, which is T1W1 and proton MR spectroscopy is useful to distinguish between HIE and normal infants, However the study presented in [20] is performed manually on MRIs. In my opinion, these two areas have been not evaluated on large datasets or fail to give a good result for the largest number of images in screening processing. To the best of our knowledge, no attempt has been made to automate the HIE diagnosis so far in the medical image processing community. Some approaches are focused on ridge detection of the vessels' centreline and rebuilt the vessel 3D structure for the detection of the sickle cell disease [10]. Some methods are used to develop the application of Hessian matrix eigenvalues and eigenvectors to find the ridge-valley inflection points and the application of eigenvalues [11]. The aforementioned methods are not automated to provide advice to medical personnel.

In this paper, a method is proposed to aid diagnosis in infants who have HIE automatically by processing their SW images. In our work, we extract features from SW images to classify babies into healthy and HIE infants. Our feature extraction technique proposed here is based on the fact that the appearance of anoxic vessels is different from the healthy vessels in that the anoxic vessels look darker than the healthy ones. Here a ridge detection method is applied to detect the vessels. A pixel on the detected ridges (vessels) is then assigned a value proportional to the eigenvalue of 
Hessian matrix in that pixel to obtain a soft ridge map of the SWI. The histogram of such a soft ridge map for each infant's SWIs is then calculated. A curve characterized by four parameters is also fit to the soft ridge histograms to avoid overfitting issues. In our method, SW images are classified based on the parameters of this curve. Our method provides a reliable diagnosis without the need for specialists to spend a great deal of time to examine SWIs. The diagnosis provided by our system is also free from human error.

\section{Methodology}

\subsection{Ridge Detection}

Ridge detection in this research is used to define the centreline of deoxygenated vessels. We apply a local median filter and Gaussian filter to remove the noise first. A median filter removes the noise and preserves most of the original features of SWI. As the SWIs are three dimensional images consisting of two dimensional slices, we, therefore, start our analysis of ridge detection for a two dimensional grey scale image. This ridge detection method is based on the retinal vessel segmentation method [1214, 18].

Let us denote $I(\mathbf{x})$ with $\mathbf{x}=(x, y)^{T}$ to be the SW image, where the first derivative of the image $I(\mathbf{x})$ in the direction perpendicular to the ridge tangent has a zerocrossing. Because images contain noise a Gaussian filter with a standard deviation of $\sigma$ is convolved with the image derivative, i.e.: [12].

$$
I\left(x_{i}\right)=\frac{\partial I(\mathbf{x}, \sigma)}{\partial x_{j}}=\frac{1}{2 \pi \sigma^{2}} \int_{\mathbf{x}^{\prime} \in \mathbb{R}^{2}} \frac{\partial e^{-\left\|\mathbf{x}-\mathbf{x}^{\prime}\right\|^{2} / 2 \sigma^{2}}}{\partial x_{j}} L\left(\mathbf{x}^{\prime}\right) d \mathbf{x}^{\prime}
$$

where $x_{j}$ is the image coordinate with respect to which the derivative is taken. The higher order derivatives could be computed by taking a higher order derivative of the Gaussian kernel.

The ridge point is detected by setting pixels corresponding to local minima (valley ridges) to -1 pixels corresponding to local maxima (hill ridges) to +1 and 0 otherwise by using the following equation in a simple ridge detector [12]:

$$
R(\mathbf{x}, \sigma)=-\frac{1}{2} \operatorname{sign}(\lambda)|\operatorname{sign}(\nabla I(\mathbf{x}+\widehat{\mathbf{v}}, \sigma) \cdot \widehat{\mathbf{v}})-\operatorname{sign}(\nabla I(\mathbf{x}-\widehat{\mathbf{v}}, \sigma) \cdot \hat{\mathbf{v}})|
$$

where the gradient operator $\nabla$ is defined as $(\partial / \partial x, \partial / \partial y)^{T}$ and $\lambda(\mathbf{x}, \sigma)$ is the largest absolute value of the eigenvalue for Hessian matrix $H$, in each pixel as calculated in Eq. (3). Eigenvalue $\lambda$ and $\widehat{\mathbf{v}}$ are then evaluated at $(\mathbf{x}, \sigma)$. Ridge map $R$ calculated in Eq. (2) contain both valley and hill ridges. Such a ridge map is known as hard ridge since the ridge location is specified in the map however the ridge magnitudes are ignored in this ridge map. 


$$
H=\left[\begin{array}{ll}
I_{x x} & I_{x y} \\
I_{x y} & I_{y y}
\end{array}\right]
$$

where $I_{a b}=\frac{\partial^{2} I}{\partial a \partial b}$.

Since we are interested in only vessels corresponding to valley ridges, we aim to construct a ridge map containing only valley ridges. SWIs of the deoxygenated infants demonstrate darker and wider vessels corresponding to deeper valleys. It is, therefore, interesting to know how deep or shallow a valley (vessel) is for the diagnosis of HIE. To this end, the absolute maximum eigenvalue of the Hessian matrix associated with each pixel on the ridge is calculated and then the corresponding pixel value in the ridge map is set to the absolute maximum eigenvalue. Otherwise, the pixel values of the ridge map in non-ridge locations are considered zero. The eigenvalues of a Hessian matrix on a ridge point is proportional to how deep the valley ridge (vessel) is, and the direction of the eigenvector on that ridge point is perpendicular to the ridge tangent [13]. The ridge map produced with the aforementioned process is known as soft ridge map as calculated by Eq. (4).

$$
R_{\text {Soft Ridge }}[\mathbf{x}]=\left\{\begin{array}{cc}
\lambda_{i}(\mathbf{x}) & \max _{i}\left(\left|\lambda_{i}(\mathbf{x})\right|\right) i=1,2 \\
0 & \text { otherwise }
\end{array}\right.
$$

where $\lambda_{1}, \lambda_{2}$ are the eigenvalues of the Hessian matrix.

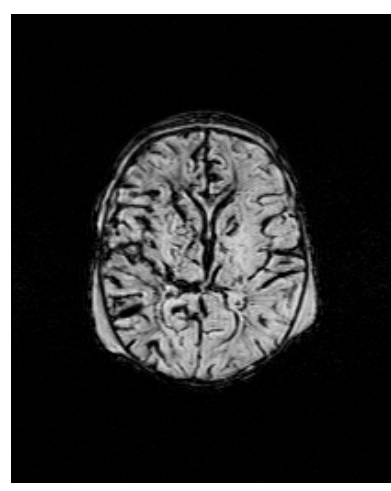

(a)

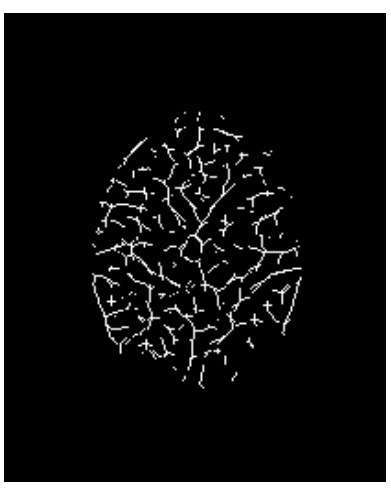

(b)

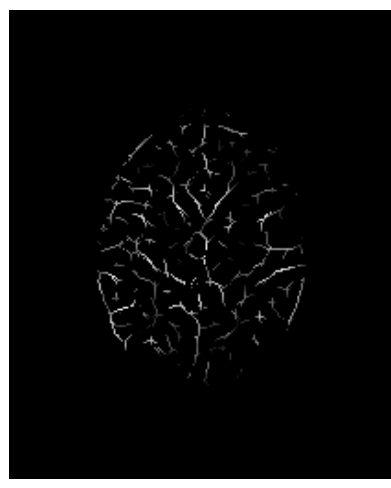

(c)

Fig. 1. Ridge maps for an SW image of an infant with HIE. (a) Original SW image smoothed with a Gaussian kernel, $\sigma=2.0$ pixels. (b) The hard ridge corresponding to local minima ridges. (c) The soft ridge map. 


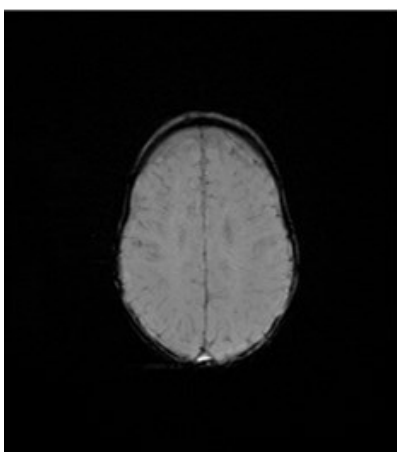

(a)

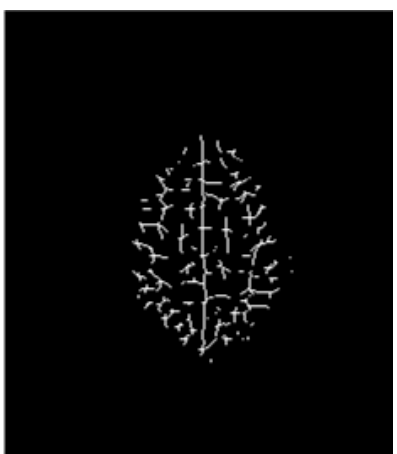

(b)

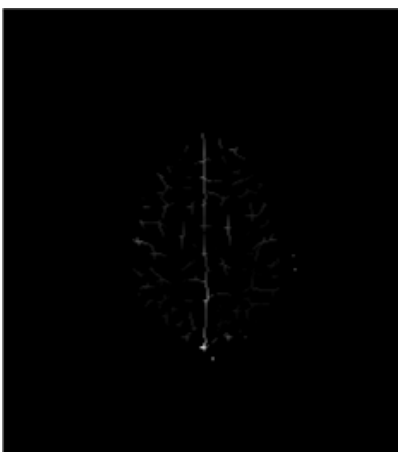

(c)

Fig. 2. Ridge maps for an SW image of a healthy infant. (a) Original SW image smoothed with Gaussian kernel, $\sigma=2.0$ pixels. (b) The hard ridge corresponding to local minima ridges. (c) The soft ridge map.

By comparing with abnormal (Fig. (1-c)) and normal (Fig. (2-c)) soft ridge maps, the soft ridge maps of an SWI indicates the presence and severity of the HIE. Therefore, the soft ridge detection is an effective method to detect and determine the presence and severity of deoxygenated brain in SWIs.

\subsection{Feature Extraction}

The deoxygenated vessels in the cerebrum of SWIs are used to produce the soft ridge maps as explained in the previous section. The histograms of soft ridge maps are initially calculated. In these histograms, we ignore the pixels with grey scale zero, because they correspond to the background and are of no importance. The soft ridge maps are calculated for all slices of SW images of an infant. Then the histogram of the soft ridge maps for all slices is computed for an infant. In fact, this histogram is considered a feature representing all slices of SWIs of an infant. Such a histogram is shown in Fig. (3-a) for an infant suffering from HIE. The histogram for a healthy baby is also depicted in Fig. (3-b). In these figures, the bins start from 0.00001 with the bin size of 0.001. In our numerical experiments, the maximum value of these histograms in our dataset is around 30. In our dataset, there are 10 healthy infants and 48 infants suffering from HIE with various scores of severity. In order to avoid overfitting problems, we fit a curve whose function is presented in Eq. (5). This function is characterised with four parameters $a, b, c, n$, i.e.:

$$
D=\frac{a}{\sqrt{x^{n}}} e^{-(b x+c / x)}
$$

where $x$ is the grey scale of the soft ridge map (the horizontal axis in Fig. 3). 


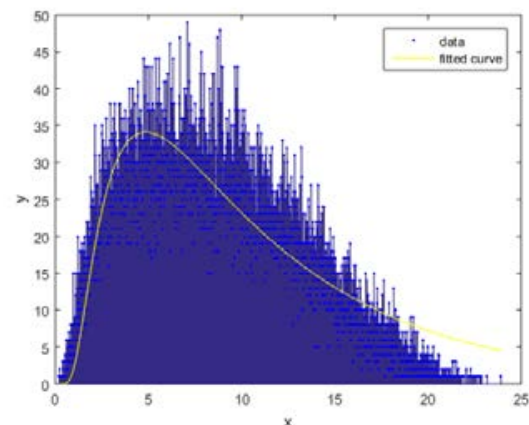

(a)

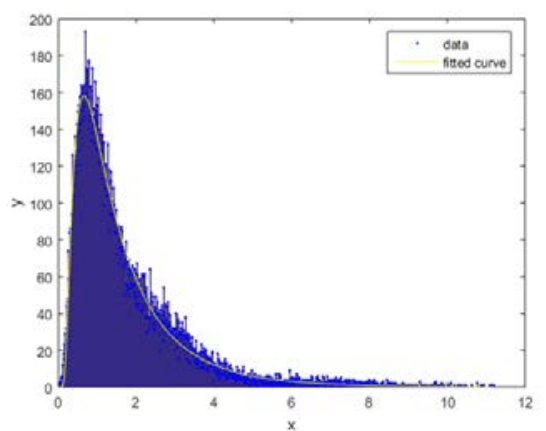

(b)

Fig. 3. (a) Histogram with a fitting curve of the infant of Fig. 1. (b) Histogram with a fitting curve of the infant of Fig. 2. The yellow curves are the fitting curving using Eq. (5).

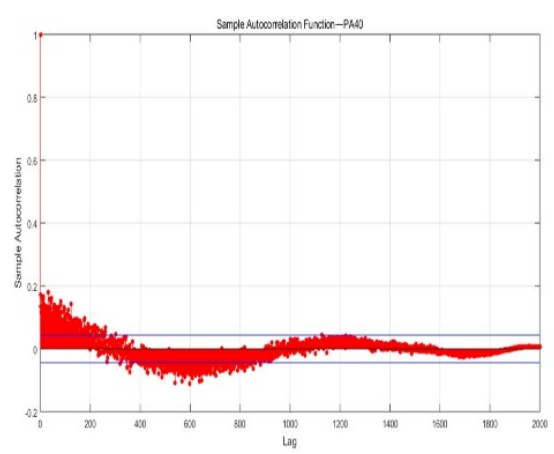

(a)

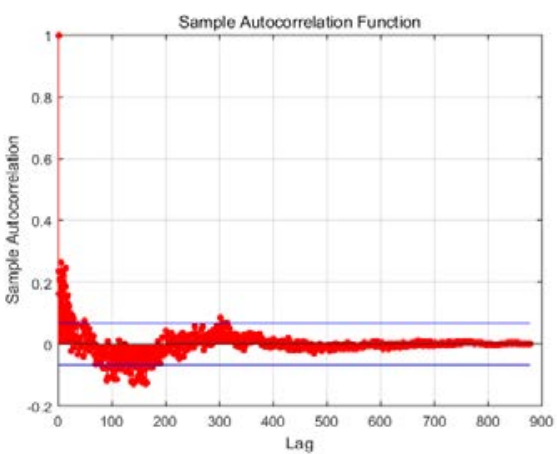

(b)

Fig. 4. The autocorrelation plot of the errors between the histogram and fitting curve. (a) The autocorrelation plot for a patient with HIE. (b) The autocorrelation plot for a healthy infant. The red points illustrate the correlation error between the histogram and the fitting curve. Two parallel lines on the both side of the coordinate axis are the error bounds, which have a default value of 2 standard deviations.

Equation (5) is fit to the histograms by using a nonlinear least square technique with $95 \%$ confidence bounds. The results of the fitting process are also presented in Fig.3 for both histograms. The autocorrelation of the errors between the actual histogram data and the fitted curve is also depicted in Fig. (4-a) can be observed from Fig.4, most of the autocorrelation data is random, however, there is a pattern inside the autocorrelation function. This is due to that fact that function proposed in Eq. (5) can be modified for a better fit until the autocorrelation function contains only random data. Since the modification of Eq. (5) is an intuitive process which is based on trial and error, we leave this for our future work. These autocorrelations are calculated with the estimation error bounds 2 standard deviations away from zero which corresponds to $95 \%$ confidence bounds. 


\section{$3 \quad$ Results}

The deoxygenated vessels in SW images are detected with the ridge detection method discussed in section 2. It is, therefore, important to evaluate the performance of our ridge detection algorithm by plotting its receiver operating characteristic (ROC) curve [15-16], before the feature extraction process. In our data set, there are over $2400 \mathrm{SW}$ 45images, which come from 58 infants and each infant dataset contains more than 40 slides. We manually detect the ridges for 200 slices of these SW images and also apply our ridge detection algorithm to these 200 slices of SW images for comparison purposes. The ridges are manually annotated to compare the annotated ridges with the ridges detected by our algorithm to measure the performance of our algorithm using ROC curves. We have applied Gaussian and local mean filters on the SW images prior to the ridge detection. The performance evaluations of our method are plotted as ROC curves shown in Fig. 5.

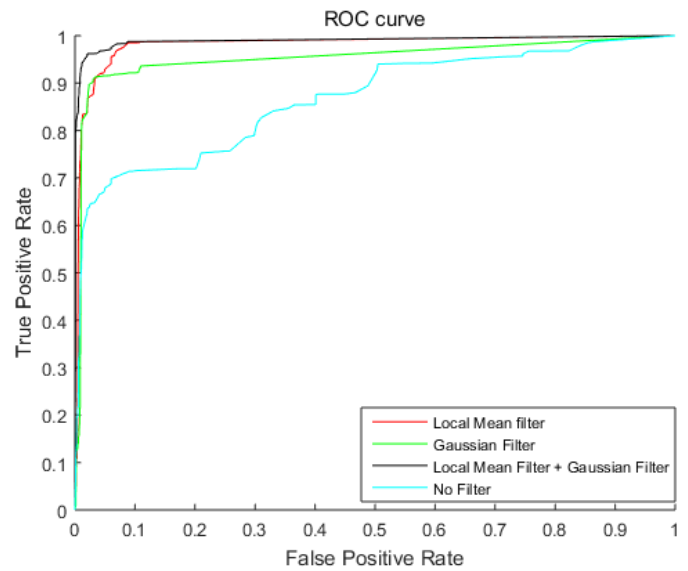

Fig. 5. ROC curves to evaluate the performance of our ridge detection algorithm when various filtering schemes are used before the ridge detection. The cyan line is the ROC curve corresponding to the case no filter is used. The green ROC curve is when the original SW images are filtered with a Gaussian kernel with the standard deviation of 2 . The red ROC curve is associated with the case where a local $3 \times 3$ mean filter is employed prior to ridge detection. The black ROC curve represents the performance of our ridge detection when both filters (Gaussian and mean filters) are applied to SW images.

The ROC curve is created by plotting the rate of the manually detected vessel points (true positive rate) against the rate of the auto-detected vessel points which are not detected by manual work (false positive rate). The cyan ROC curve corresponds to the case where no filter is applied to the original SW images. The green ROC curve is associated with the case where the Gaussian filter with standard deviations of 2 is applied to the SW images prior to the ridge detection. The red ROC curve is obtained by employing a local $3 \times 3$ mean filter. Finally, the black ROC curve corresponds to the case where both Gaussian and the local mean filters are employed. As can be seen 
from Fig. 5, the best performance is achieved, when the Gaussian filter is applied before the local mean filter on SWI slices prior to ridge detection process.

In the next experiment, we would like to classify the infants whose SWIs are in our dataset, into two groups: healthy infants and infants with HIE. Initially, the histograms of the soft ridge maps are recalculated with 24 bins. Therefore there are 24 features (parameters) for each histogram. These features (parameters) are then fed into various kinds of classifiers presented in Table 1.

Table 1. Classification accuracy with 24-bin histograms to represent SWIs

\begin{tabular}{|c|c|c|c|}
\hline $\begin{array}{c}\text { Classification } \\
\text { Method }\end{array}$ & $\begin{array}{r}\text { Branch I } \\
\text { the } \mathrm{Cr}\end{array}$ & & Accuracy Result \\
\hline \multirow{4}{*}{$k N N$} & \multicolumn{2}{|c|}{$k=1$} & $89.66 \%$ \\
\hline & \multicolumn{2}{|c|}{$k=3$} & $91.83 \%$ \\
\hline & \multicolumn{2}{|c|}{$k=5$} & $91.83 \%$ \\
\hline & \multicolumn{2}{|c|}{$k=7$} & $89.66 \%$ \\
\hline \multirow{5}{*}{ SVM } & \multicolumn{2}{|c|}{ Gaussian Kernel } & $87.93 \%$ \\
\hline & \multicolumn{2}{|c|}{ Linear } & $86.21 \%$ \\
\hline & \multirow{3}{*}{$\begin{array}{c}\text { Polynomial } \\
\text { (Kernel Order: } p \text { ) }\end{array}$} & $p=2$ & $94.83 \%$ \\
\hline & & $p=3$ & $93.10 \%$ \\
\hline & & $p=4$ & $91.83 \%$ \\
\hline
\end{tabular}

As can be seen from this table, the SVM classifier with a polynomial kernel with order 2 produces the best classification accuracy of $94.83 \%$ and the cross-validation rate of approximately $5 \%$. It is important to note that every infant in our dataset is already diagnosed by specialists to be either healthy or with HIE illness. To calculate, the classification accuracies presented in Table 1, a leave-one-out strategy in which the data of an infant is taken out as a test sample without any knowledge of the specialists' diagnosis, is employed. Leave-one-out strategy is a type of cross validated, which the predicted infant's data is picked out of the validation dataset (soft ridge map dataset of whole infants).

Having fit the curve (Fig. 5) to the histograms of the SWIs in our dataset, every infant is represented by a feature vector containing the values of the four parameters a, $\mathrm{b}$, c and $\mathrm{n}$. These vector features are then fed to $k \mathrm{NN}$ and SVM classifiers with various classification parameters and various kernels to produce the accuracy classification results presented in Table 2. As explained before for Table 1, a leave-one-out strategy is also employed here to calculate the classification accuracies. The best classification result achieved with the curve fitting method is $91.38 \%$ which is with an SVM classifier with a polynomial kernel of order 3 . It is important to note that the fitting curve method may be characterised with a slightly lower accuracy result but it avoids the problems related to overfitting issues from which the first method associated with 24 bin histograms, may suffer. 
Table 2. Classification accuracy with parameters of equation (5) chosen as features to represent SWIs

\begin{tabular}{|c|c|c|c|}
\hline $\begin{array}{l}\text { Classification } \\
\text { Method }\end{array}$ & \multicolumn{2}{|c|}{$\begin{array}{l}\text { Branch Method and } \\
\text { the Coefficient }\end{array}$} & Accuracy Result \\
\hline \multirow{4}{*}{$k N N$} & \multicolumn{2}{|c|}{$k=1$} & $84.48 \%$ \\
\hline & \multicolumn{2}{|c|}{$k=3$} & $89.66 \%$ \\
\hline & \multicolumn{2}{|c|}{$k=5$} & $86.20 \%$ \\
\hline & \multicolumn{2}{|c|}{$k=7$} & $84.48 \%$ \\
\hline \multirow{5}{*}{ SVM } & \multicolumn{2}{|c|}{ Gaussian Kernel } & $87.93 \%$ \\
\hline & \multicolumn{2}{|c|}{ Linear } & $81.03 \%$ \\
\hline & \multirow{3}{*}{$\begin{array}{c}\text { Polynomial } \\
\text { (Kernel Order: } p \text { ) }\end{array}$} & $p=2$ & $87.93 \%$ \\
\hline & & $p=3$ & $91.38 \%$ \\
\hline & & $p=4$ & $84.48 \%$ \\
\hline
\end{tabular}

To evaluate the classifiers' performance, the precision (specificity) and recall (sensitivity) values of the HIE infants is presented in Table 3 . Table 3 is clearly and directly to show the advantages of our classification features and classifier, which shows the sensitivity and specificity of this automatic algorithm. The method presented in [21], has a sensitivity and specificity of 0.72 and 0.99 respectively. Therefore the performance of our algorithm is better than the method presented in [21] in terms of sensitivity and specificity values.

Table 3. The precision and recall values for the classifiers employed in Table 2.

\begin{tabular}{cccc}
\hline $\begin{array}{c}\text { Classification } \\
\text { Method }\end{array}$ & $\begin{array}{c}\text { Branch Method and } \\
\text { the Coefficient }\end{array}$ & $\begin{array}{c}\text { Precision } \\
\text { Value }\end{array}$ & $\begin{array}{c}\text { Recall } \\
\text { Value }\end{array}$ \\
\hline \multirow{3}{*}{$k$ kNN } & $k=1$ & $92 \%$ & $93.88 \%$ \\
& $k=3$ & $91.84 \%$ & $91.84 \%$ \\
$k=5$ & $90.57 \%$ & $100 \%$ \\
$k=7$ & $90.38 \%$ & $100 \%$ \\
\hline \multirow{4}{*}{ SVM } & Gaussian Kernel & $87.27 \%$ & $100 \%$ \\
& Linear & $85.71 \%$ & $100 \%$ \\
& Polynomial $\quad p=2$ & $94.83 \%$ & $95.92 \%$ \\
& (Kernel Order: $p$ ) $\quad p=3$ & $93.10 \%$ & $92.31 \%$ \\
& $p=4$ & $91.83 \%$ & $92 \%$ \\
\hline
\end{tabular}




\section{$4 \quad$ Conclusion and Future Work}

The method in this paper presents an automatic diagnosis of HIE based on SWIMRIs. Unlike the previous ridge detection methods, the eigenvalues of ridge pixels intuitively determine if an infant suffers from HIE or not. The image containing the eigenvalues of the Hessian matrix for all ridge pixels is known as the soft ridge map. The soft ridge maps consist of the vessels detected as ridges with eigenvalues of the Hessian matrix calculated for each ridge pixel. In the method proposed here, we find the features as histograms of soft ridge maps to represent each infant for classification. To reduce the dimensionality of our feature space, we have used two techniques: 1) simply reducing the number is bins into 24 and 2) exploiting the parameters of the curve fitted to the histograms. In the second technique, after the soft ridge detection, the histogram of each patient's soft map is represented by a curve. This curve has been optimally fitted to that histogram. Such a curve is described by four parameters which are used as features for classification stage. Then these features (each consisting of four parameters) are used to classify infants as healthy or a baby suffering from HIE, with $k N N$ and SVM classifiers. The SVM classifier with polynomial kernel of order 2 presents the best accuracy rate of $91.38 \%$. A leave-one-out strategy is employed for the measurements of classification accuracies. Our automatic classification method can, therefore, produce reliable diagnosis without the specialists' interventions and inspections. As a result, our method is faster than a specialist for diagnosis, is not prone to human errors and would save the specialists' time taken for diagnosis. Our method could increase the prognostic value of these SWIs, thereby it improves the management of the illness as well as elucidates the disease mechanisms. This method can also be extended to estimate different MR scores $[17,19]$ assigned by specialists to HIE infants. Such an extension is an interesting topic for our future work.

\section{$5 \quad$ Acknowledgment}

The research database is provided by Dr. Brigitte Vollmer and Dr. Angela Darekar, Southampton General Hospital.

\section{Reference}

1. Perlman, Jeffrey M. "Summary proceedings from the neurology group on hypoxicischemic encephalopathy." Pediatrics 117.Supplement 1 (2006): S28-S33.

2. Fatemi, Ali, Mary Ann Wilson, and Michael V. Johnston. "Hypoxic-ischemic encephalopathy in the term infant." Clinics in perinatology 36.4 (2009): 835-858.

3. James, Anitha, and Vaishali Patel. "Hypoxic ischaemic encephalopathy." Paediatrics and Child Health 24.9 (2014): 385-389.

4. Shankaran, Seetha, et al. "Effect of depth and duration of cooling on deaths in the NICU among neonates with hypoxic ischemic encephalopathy: a randomized clinical trial." Jama 312.24 (2014): 2629-2639. 
5. Friberg, Hans, and Tobias Cronberg. "Hypoxic-Ischemic Encephalopathy." Seminars in neurology. Vol. 37. No. 01. Thieme Medical Publishers, 2017.

6. OU, Chang-xue, Fei-ying XIAO, and Duo-cheng SUN. "The Value of Diffusion Weighted Imaging in the Early Diagnosis and Prognos-tic Evaluation of Neonatal HIE." Chinese Journal of CT and MRI 1 (2013): 011.

7. Bozzao, Alessandro, et al. "Diffusion-weighted MR imaging in the early diagnosis of periventricular leukomalacia." European radiology 13.7 (2003): 1571-1576.

8. Massaro, An Nguyen, et al. "Short-term outcomes after perinatal hypoxic ischemic encephalopathy: a report from the Children's Hospitals Neonatal Consortium HIE focus group." Journal of Perinatology 35.4 (2015): 290-296.

9. Vergales, Brooke D., et al. "Depressed heart rate variability is associated with abnormal EEG, MRI, and death in neonates with hypoxic ischemic encephalopathy." American journal of perinatology 31.10 (2014): 855-862.

10. Winchell, Adam M., et al. "Evaluation of SWI in children with sickle cell disease." American Journal of Neuroradiology 35.5 (2014): 1016-1021. Hladůvka, Jiří, Andreas König, and Eduard Gröller. "Exploiting eigenvalues of the Hessian matrix for volume decimation." (2001).

11. Bofill, Josep Maria, and Wolfgang Quapp. "Analysis of the Valley-Ridge inflection points through the partitioning technique of the Hessian eigenvalue equation." Journal of Mathematical Chemistry 51.3 (2013): 1099-1115.

12. Staal, Joes, et al. "Ridge-based vessel segmentation in color images of the retina." IEEE transactions on medical imaging 23.4 (2004): 501-509.

13. Annunziata, Roberto, et al. "Leveraging multiscale hessian-based enhancement with a novel exudate inpainting technique for retinal vessel segmentation." IEEE journal of biomedical and health informatics 20.4 (2016): 1129-1138.

14. Wang, Yangfan, et al. "Retinal vessel segmentation using multiwavelet kernels and multiscale hierarchical decomposition." Pattern Recognition 46.8 (2013): 2117-2133.

15. Hajian-Tilaki, Karimollah. "Receiver operating characteristic (ROC) curve analysis for medical diagnostic test evaluation." Caspian journal of internal medicine 4.2 (2013): 627.

16. Hand, David J. "Measuring classifier performance: a coherent alternative to the area under the ROC curve." Machine learning 77.1 (2009): 103-123.

17. Barkovich, A. James, et al. "Prediction of neuromotor outcome in perinatal asphyxia: evaluation of MR scoring systems." American Journal of Neuroradiology 19.1 (1998): 143149.

18. Chen, Da, and Laurent D. Cohen. "Automatic Tracking of Retinal Vessel Segments using Radius-Lifted Minimal Path Method." MIUA 2015. 2015.

19. Kitamura, Gene, et al. "Hypoxic-ischemic injury: utility of susceptibility-weighted imaging." Pediatric neurology 45.4 (2011): 220-224.

20. Guo, L., Wang, D., Bo, G., Zhang, H., Tao, W., \& Shi, Y. (2016). Early identification of hypoxic-ischemic encephalopathy by combination of magnetic resonance (MR) imaging and proton MR spectroscopy. Experimental and Therapeutic Medicine, 12(5), 2835-2842. http://doi.org/10.3892/etm.2016.3740

21. Murphy, K., van der Aa, N. E., Negro, S., Groenendaal, F., de Vries, L. S., Viergever, M. A., Boylan, G. B., Benders, M. J. N. L. and Išgum, I. (2017) 'Automatic quantification of ischemic injury on diffusion-weighted MRI of neonatal hypoxic ischemic encephalopathy', NeuroImage: Clinical, 14, pp. 222-232. doi:10.1016/j.nicl.2017.01.005 